\title{
Ausencia congénita de vagina
}

\author{
William Onatra H*; Clara Arteaga**; Orlando Pérez ${ }^{* * *}$
}

\begin{abstract}
RESUMEN: Se presentan los principales aspectos de la historia, frecuencia, embriología y genética de la ausencia congénita de vagina.
Se analizaron 850 historias de la clínica de Endocrinología del Instituto Materno Infantil durante el período de 1970 a 1988 , encontrándose 45 casos $(4.1 \%)$ de agenesia de vagina, proponiendo los autores 4 categorías con los siguientes criterios:

I. Ausencia congénita de vagina con disgenesia gonadal (XX o XY). 4 casos-46XX (8.6\%) $12 X Y(26 \%)$.

II. Ausencia congénita de vagina con gónada normal (síndrome de Rockitansky Kuster Hauser) 19 pacientes (41.4\%).

III. Ausencia congénita de vagina con anomalías de fusión de ductus mulerianos (3 pacientes) 3 casos (6.6\%).

IV. Ausencia congénita de vagina con útero y gónada normal (tabiques, longitudinal transverso) 8 pacientes. 8 casos (17.4\%).

Se propone el siguiente manejo: Grupo I, terapia de suplencia y adopción si la paciente desea. Grupo II, neovagina y si desea embarazo madre sustituta. Grupo III, insiminación intrauterina. Plastia utrina según el caso. Grupo IV, utilizar la cánula endotraquial para evitar la histerectomía.
\end{abstract}

PALABRAS CLAVES: Neovagina, plastia vaginal, ausencia vagina.

SUMMARY: We show the major topics in the history, frecuency, embriology and genetics of the congenital lack of vagina.

We analyzed 850 histories from the endocrinology clinic in the Materno Infantil Institute during the period between 1970 to 1988.

We found 45 cases $(4.1 \%)$ of congenital lack of vagina. The autors propose fourth categories as follow:

I. Congenital lack of vagina with gonadal disgenesy (xx o xy) 4 cases $46 \times x(8.6 \%)-12$ xy cases $(26 \%)$.

II. Congenital lack of vagina with normal gonades (Rockitansky Kuster Hauser Sind) 19 patients (41.4\%).

III. Congenital lack of vagina with fussion anomaly of mullerian ductus ( 3 patients) 3 cases $6.6 \%$ ).

IV. Congenital lack of vagina with normal uterus and gonades (transverse o longitudinal septums) 8 patients $(17.4 \%)$.

We propose the following manage:

I. Suplency terapy and adoption, if the patient desine childs.

II. Neovagina. If the patient want a child think in substitute mother.

III. Intrauterine insemination o uterine surgery if there is possible.

IV. Use an endothraqueal canule for missing histerectomy.

Conclusion: If we could classified the congenital lack of vagina we could choose the right manage and prevent the obstetric future of owns patients.

KEY WORDS: Neovagina, uterine surgery, lack of vagina.

La atresia o ausencia de vagina puede formar parte de varios estados intersexuales, presentarse como anomalía aislada en presencia de un útero normal o asociarse a otras anomalías de los ductos mullerianos.

\section{Historia}

La primera referencia sobre ausencia congénita aparece en 1572 en una descripción hecha por Realdus Columbus, discípulo de Vesalio (2); el reconocimiento de su asociación con un útero anómalo o ausente fue la base de otros trabajos. Mayer (1), en 1829 describió la ausencia congénita de vagina en mortinatos femeninos asociada con múltiples defectos congénitos. Rockitansky (2), en 1838 y Kuster (3), en 1910 describieron independientemente una entidad consistente en ausencia de vagina con útero rudimentario o bidelfo y ovarios normales, asociado a anomalías renales y esqueléticas. Finalmente, en 1961 Hauser (4) precisó datos sobre la frecuencia de este desorden y describió todo el espectro de las

\footnotetext{
* Universidad Nacional de Colombia. Profesor Asistente. Departamento de Obstetricia y Ginecología.

** Profesora Asistente. Departamento de Morfología. Seción Genética.

*** Profesor Asistente. Departamento de Cirugía. Sección Cirugía

Plástica. Uni versidad Nacional de Colombia. Bogotá.
}

anomalías asociadas y en la actualidad se conoce como síndrome de Mayer-Rockitansky-Kuster-Hauser (5, 10, 30, 31).

\section{Frecuencia}

La frecuencia de la agenesia útero-vaginal en los estudios poblacionales varía según los autores. David (7) refiere una incidencia de una paciente por cada 4.000 a 5.000 mujeres que acuden a la consulta de ginecología. Fore y col. (9) hacen referencia a grupos de pacientes con amenorrea primaria asociada a ausencia congénita de vagina variando la frecuencia entre 1 por 1.494 hasta 1 por 80.000 casos. Desde el punto de vista de la consuita por amenorrea primaria según Ross y Vande Wiele (10) este defecto representa la 2a causa después de la disgenesia gonadal.

\section{Embriología}

Los ductos internos de los embriones de ambos sexos dependen en último término del llamado sistema de riñón mesonéfrico, derivado del mesodermo. Hacia la semana quinta de desarrollo se encuentra una estructura llamada riñón y otra conocida como ducto mesonéfrico o wolffiano, éste último conectado con el seno urogenital. Hacia la semana sexta se inicia el desarrollo de una estructura ductal 
paralela a la anterior, llamada ducto paramesonéfrico o ducto mulleriano. Dos porciones pueden distinguirse en esta estructura: una anterior o cefálica derivada del epitelio celómico y otra caudal derivada del ducto mesonéfrico e íntimamante ligada a él en su desarrollo y que desemboca en el seno urogenital. La unión entre estos pares de ductos es de tal magnitud que no pueden formarse ductos mullerianos en ausencia de ductos wolffianos.

El terminal cefálico de los ductos mullerianos da origen a las trompas de falopio y al útero, la porción caudal al tercio superior de vagina. La unión del terminal caudal con el seno urogenital, estimula la proliferación celular y por consiguiente la elongación de esta estructura dando lugar a la llamada "placa vaginal" hacia la semana octava de desarrollo. Esta estructura sólida, por lisis celular inicia su canalización para formar la vagina propiamente dicha hacia la doceava semana (Gráfica No. 1).

\section{DESARROLLO DE VAGINA}

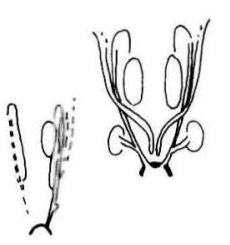

MESONEFROS

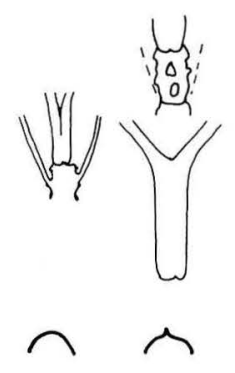

S. UROGENITAL

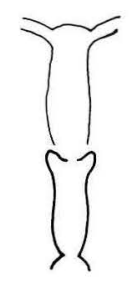

CAVITACION
Cuando el desarrollo es alterado por cualquier factor antes de la fusión de los ductos mullerianos, ocurre una agenesia total de derivados del muller, trompas, útero y tercio superior de vagina $(15,16,17)$.

En la agenesia útero vaginal la formación de la porción cefálica de los ductos mullerianos ocurre de manera normal encontrándose por lo tanto trompas normales. Sin embargo, si el desarrollo subsiguiente de la porción caudal a lo largo de los ductos mullerianos se altera, produce agenesia de útero y de tercio superior de vagina. Se sugiere que una alteración primaria a nivel de los ductos mullerianos, produce anomalía útero-vaginal y anomalías renales si se tiene en cuenta que este ducto es también el primordial ducto metanéfrico, origen del sistema renal (Gráfica No. 1).

Las anomalías esqueléticas pueden explicarse por el origen mesodérmico común entre el mesonefros y los somitas adyacentes que darán origen al esqueleto y los cuales se diferencian simultáneamente en el embrión $(5,19,20)$. En la agenesia del tercio inferior de vagina donde consideramos también el himen imperforado, la alteración deberá encontrarse a nivel del seno urogenital y por lo tanto también puede asociarse a anomalías renales o esqueléticas.

Como los ductos mullerianos son originalmente órganos pares, su fusión y canalización posterior formarán el tercio superior de vagina, útero y trompas. Una alteración en la fusión dará lugar a dos hemiúteros. En otras ocasiones uno de los ductos mullerianos no contribuye a la formación del útero y sólo produce un cuerpo rudimentario. Clínicamente se han descrito una serie de variedades como el útero unicorne, útero arcuato (depresión media en el fondo del útero), uterus subseptus (persistencia de un septo en la porción superior del útero), uterus septus (septo uterino completo), útero bicorne (dos cavidades separadas desembocando en un sólo cervix o dos cervix separados), útero bidelfo (dos cavidades uterinas desembocando en dos cuellos y en dos vaginas completamente septadas).

\section{Cuadro clínico}

Como se mencionó anteriormente la agenesia vaginal puede presentarse como una anomalía aislada o formando parte de un grupo complejo de malformaciones. En cualquier caso su motivo de consulta es la amenorrea primaria acompañada o no de dolor abdominal cíclico. La aparición de las características sexuales secundarias procede de manera normal y a la edad esperada excepción hecha de la menarquía. El examen ginecológico muestra genitales externos femeninos normales.

Excepto en los casos XY en los cuales son ambiguos. Se puede apreciar la presencia de un tabique, un fondo de saco ciego, una hipoplasia o una ausencia completa de vagina. El tacto rectal y la ecografía pélvica pueden informar sobre las características del útero; excepcionalmente por ecografía se puede detectar su ausencia, el útero bicorne o didelfo. La laparoscopia es confirmatoria de la presencia o no de útero, trompas, ovarios y sus características.

Las evaluaciones endocrinológicas se encuentran siempre dentro de límites normales a excepción de la asociada a disgenesia gonadal XX o XY, en las que la FSH y LH están elevadas y el estradiol bajo. La testosterona suele elevarse al inicio de la pubertad en los casos de testículo feminizante. Los estudios cromosómicos son imprescindibles con el fin de descartar las variedades mosaico y determinar los casos $\mathrm{XY}$.

Griffin (10) en 1976 encuentra en su revisión de 14 pacientes con agenesia de vagina y alteración del útero que una tercera parte de ellas (32.8\%) presentan anomalías renales de tipo agenesia o ectopía unilateral (75\%), riñón en herradura $(5 \%)$ y otras anomalías en los sistemas colectores o malrotaciones $(20 \%)$. Fore (9) encuentra una frecuencia de $47 \%$ de alteraciones urológicas en su serie de 43 , pacientes con amenorrea primaria y ausencia congénita de vagina. En el $12 \%$ de éstas se presentaron anomalías esqueléticas a nivel de columna (66\%), de miembros y costillas (34\%) $(10,20)$.

\section{Genética}

La mayoría de las pacientes con agenesia útero-vaginal presentan cariotipos normales femeninos. Grover y col. (11) en 1970 en revisión sobre 15 pacientes con aplasia de ductos mullerianos, encuentra constitución cromosómica 46XX normal en 14 casos y sólo un caso 46XY correspondiendo a un síndrome de feminización testicular. Existen reportes aislados que informan sobre anomalías cromosómicas en forma de mosaico 45X0/46XX y 46XX/47XXX (10). 
En la agenesia útero-vaginal se señala una ocurrencia esporádica. No obstante algunos autores informan casos familiares como Anger y col. Quienes describieron una familia con tres hermanas con ausencia congénita de vagina en las cuales no se practicaron estudios cromosómicos (10). Jones y col. (12) en 1972 reportaron agenesia congénita de vagina con útero rudimentario y ovarios normales en dos hermanas cuyos estudios cromosómicos fueron 46XX. Griffin y col. (10) en 1976 describieron una familia con dos hermanas afectadas con agenesia de vagina y una segunda familia en la cual una de ellas presentó agenesia de vagina, agenesia renal bilateral y escoliosis severa, la hermana presentó escoliosis aislada y una tía materna de las pacientes un útero bidelfo. Verp y col. (11) en 1983 describieron 3 familias donde varios miembros presentaban anomalías de fusión en los ductus mullerianos (útero bicorne o didelfo con o sin anomalías septales en vagina). Carson y col. (6) en 1983 realizaron estudios familiares a 23 casos con aplasia de ductos mullerianos, no encontrando pariente cercano afectado. Elías y col. (8) en 1984 realizaron estudios similares en 24 casos y sólo se halló una hermana con anomalía uterina.

Todas estas referencias hacen pensar que en algunos pocos casos puede existir un origen genético, sin embargo en la mayoría de los casos la anomalía se presenta en forma esporádica y sin historia familiar relacionada. Dos grupos muy importantes de pacientes con alteraciones de vagina y genitales internos masculinos lo constituyen el síndrome de feminización testicular y el pseudohermafroditismo masculino $(15,17,28)$. En el primer caso se encuentran testículos normales, constitución cromosómica 46XY, pero fenotipo externo femenino. La causa de esta lesión es una anomalía de los receptores androgénicos por una mutación génica en el cromosoma $\mathrm{X}$, por lo tanto constituyen un grupo aparte claramente definido como recesivo ligado al cromosoma $\mathrm{X}$. En el segundo caso, el pseudohermafroditismo masculino, los pacientes tienen desarrollo normal pero sus genitales externos son ambiguos con todas las variables posibles, la gónada es disgenética o elabora andrógenos en forma insuficiente, éstos se producen después de que ha pasado la sensibilidad de las estructuras sexuales. Cromosómicamente son XY.

\section{Material y métodos}

Se analizaron 850 historias clínicas de la consulta de Endocrinología ginecológica del Instituto Materno Infantil de Bogotá, durante el período de 1970-1988. Se seleccionaron| los casos de pacientes con alteraciones de diferenciación sexual y entre estos, los defectos congénitos de vagina. Se analizó la historia clínica en lo relacionado con su motivo de consulta, antecedentes familiares de malformaciones similares, antecedentes maternos de ingestión de hormonas durante el primer trimestre del embarazo de la paciente. Examen físico, peso, talla, desarrollo de las características sexuales secundarias, características de vagina. Exámenes paraclínicos: cariotipo, ecografía, (ginecografía), radiografía de columna, urografía, laparoscopia, laparotomía, tipo de tratamiento médico y/o quirúrgico aplicado y resultado.

Para la clasificación de las alteraciones de diferenciación sexual se tuvo en cuenta la propuesta por Samuel Yen (15) y aceptada por otros autores (16-18). En relación con la ausencia congénita de vagina inicialmente tomamos la de Buttran (19) y la de Rock (20). Tabla No. 1, pero dados los hallazgos de la presente investigación proponemos una modificación que puede ser útil de acuerdo con los hallazgos clínicos.

\section{Tabla No. 1}

CLASIFICACIONES DE ALTERACIONES DEL MULLER SEGUN V.C. BUTTRAM (19) Y H. JONES-J. ROCK (20)

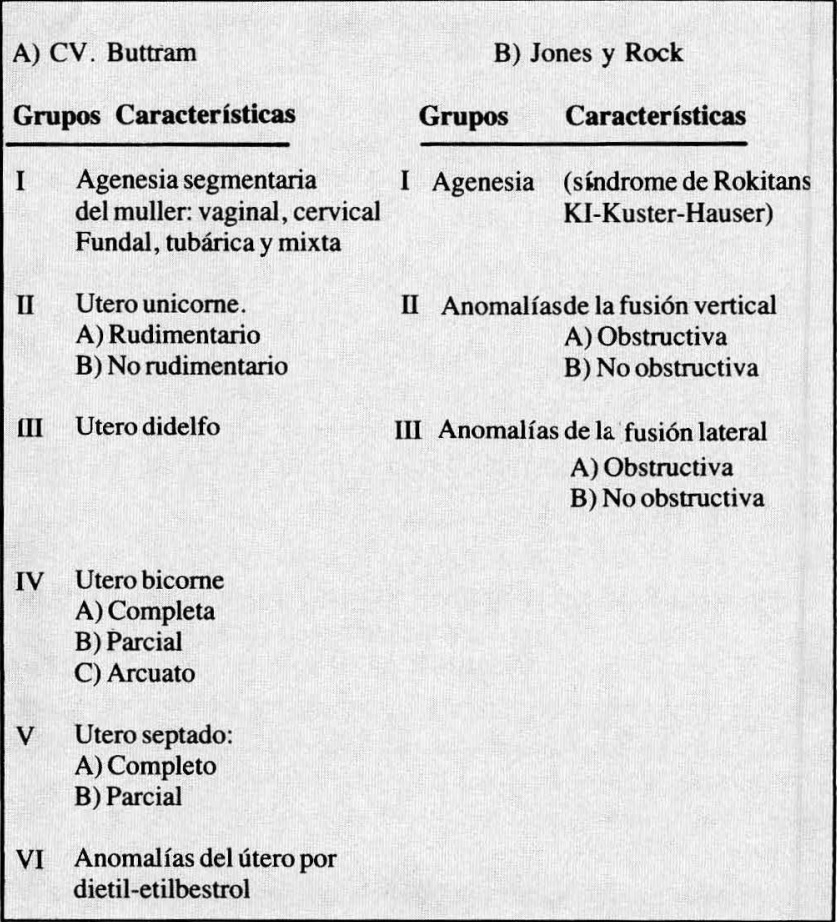

De acuerdo con la evolución embriológica y las formas como clínicamente hemos encontrado la ausencia congénita de vagina proponemos: Grupo I: las asociadas a la disgenesia gonadal. Grupo II: síndrome de Rockitansky clásico. Grupo III: el asociado a las alteraciones de fusión de muller. Grupo IV: las alteraciones del tercio superior y tabiques longitudinales o transversos. Nos parece que esta clasificación además de ser didáctica se ajusta a la clínica y engloba tanto a la de los Doctores Buttran y Jones-Rock. (Tabla No. 2, Gráfica No. 2).

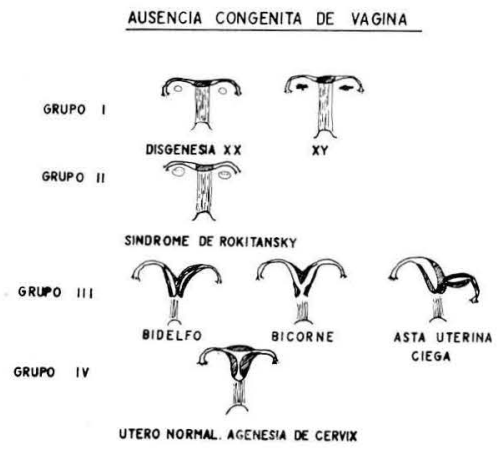


Tabla No. 2

PROPUESTA DE CLASIFICACION EN AUSENCIA CONGENITA DE VAGINA

\begin{tabular}{|c|c|c|}
\hline Grupo & & Malformación \\
\hline Grupo I & $\begin{array}{l}\text { A) } \\
\text { B) }\end{array}$ & $\begin{array}{l}\text { Ausencia congénita de vagina con disgenesia gonadal } \\
\text { Disgenesia gonadal } 46 \mathrm{XX} \\
\text { Disgenesia gonadal } 46 \mathrm{XY}\end{array}$ \\
\hline Grupo II & & $\begin{array}{l}\text { Ausencia congénita de vagina con gónada normal } \\
\text { (síndrome de Rockitansky-Kuster-Hauser) }\end{array}$ \\
\hline Grupo III & & $\begin{array}{l}\text { Ausencia congénita de vagina con anomalías de } \\
\text { fusión de ductus mullerianos. }\end{array}$ \\
\hline Grupo IV & & $\begin{array}{l}\text { Ausencia congénita de vagina con útero/gónada normal } \\
\text { (tabique longitudinal o transverso). }\end{array}$ \\
\hline
\end{tabular}

\section{Resultados}

De las 850 pacientes atendidas en la consulta de endocrinología ginecología del IMI, se encontraron 93 casos $(10.9 \%)$ con alteraciones de diferenciación sexual en donde las alteraciones del muller, entre las cuales incluimos la ausencia congénita de vagina, ocupa el segundo lugar con 35 casos $(37.6 \%)$ después de la disgenesia gonadal con 41 casos $(44 \%)$. Tabla No. 3 .

Tabla No. 3

ALTERACIONES DE DIFERENCIACION SEXUAL EN EL IMI. 1970-1988

\section{CLASIFICACION}

\begin{tabular}{|lcc|}
\hline Grupo & N & $\%$ \\
\hline Disgenesia gonadal & 16 & 17.2 \\
Disgenesia gonadal XX & 12 & 12.9 \\
Síndrome de Turner & 13 & 13.9 \\
& 13 & 13.9 \\
Hipoplasia gonadal & 4 & 4.3 \\
Pseudohermafroditismo masculino & 35 & 37.6 \\
Pseudohermafroditismo femenino & 93 & 100.0 \\
Anomalías del Muller & \\
\hline Total & 93 \\
\hline
\end{tabular}

De acuerdo con la clasificación propuesta (Tabla No. 2) incluimos en el Grupo I, 11 casos con constitución cromosómica XY y que clásicamente no aparecen incluidos en las alteraciones de fusión del muller, pero cursan con hipoplasia o agenesia vaginal y a quienes se les debe hacer una plastia de genitales incluida la neovagina. Por esta razón el número total de casos tratados con ausencia congénita de vagina es de 44 (5.2\%). Tabla No. 4.

Las características tanto clínicas como paraclínicas de los diferentes grupos aparecen en las tablas siguientes.

En el grupo IA ubicamos cuatro pacientes que presentaron hipoplasia vaginal acompañada de disgenesia gonadal XX confirmada por laparoscopia. Estas pacientes consultaron por amenorrea primaria y presentaron al examen físico hipodesarrollo de los carácteres sexuales secundarios, dos casos con fondo de saco ciego y dos casos con agenesia vaginal. Un solo caso asociado a ptosis renal derecha. Tabla No. 5 .

Se coloca a estas pacientes en un grupo particular, puesto que en la disgenesia gonadal de causa genética no se encuentra una hipoplasia vaginal como parte del cuadro clínico. En
Tabla No. 4

DISTRIBUCION DE LA AUSENCIA CONGENITA DE VAGINA -PROPUESTA DE NUEVA CLASIFICACION-

\begin{tabular}{|llrr|}
\hline Grupo & Malformación & N & \multicolumn{1}{c|}{$\%$} \\
\hline Grupo I & Ausencia congénita de vagina con disgenesia gonadal \\
& $\begin{array}{l}\text { A) Disgenesia gonadal 46XX } \\
\text { B) Disgenesia gonadal 46XY }\end{array}$ & 4 & 8.6 \\
Grupo II & $\begin{array}{l}\text { Ausencia congénita de vagina } \\
\text { gónada normal -síndrome de } \\
\text { Rockitansky- }\end{array}$ & 12 & 26.0 \\
Grupo III & $\begin{array}{l}\text { Ausencia congénita de vagina/ } \\
\text { fusión de ductos mullerianos }\end{array}$ & 19 & 41.4 \\
Grupo IV & $\begin{array}{l}\text { Ausencia congénita de vagina/ } \\
\text { útero y gónada normal (tabique longitudinal o } \\
\text { transverso, himen } \\
\text { imperforado). }\end{array}$ & 3 & 6.6 \\
\hline Total & & 17.4 \\
\hline
\end{tabular}

este grupo el tratamiento consistió básicamente en terapia hormonal de reemplazo y reconstrucción quirúrgica de la vagina. En dos casos se espera que inicien relaciones sexuales para practicar vaginoplastia.

Tabla No. 5

GRUPO IA. AUSENCIA CONGENITA DE VAGINA/DISGENESIA GONADAL XX

\begin{tabular}{|c|c|c|c|c|c|}
\hline \multicolumn{6}{|c|}{ 1.A: Antecedentes } \\
\hline $\begin{array}{l}\mathrm{n} \\
4\end{array}$ & \multicolumn{2}{|c|}{$\begin{array}{l}\text { Motivo de } \\
\text { Consulta } \\
\text { Amenorrea 1ria. }\end{array}$} & $\begin{array}{l}\text { Familiares } \\
\text { Neg. }\end{array}$ & \multicolumn{2}{|c|}{$\begin{array}{l}\text { Personales } \\
\text { Neg. }\end{array}$} \\
\hline \multicolumn{6}{|c|}{ 1.A: Cuadro clínico } \\
\hline $\begin{array}{l}n \\
4\end{array}$ & $\begin{array}{l}\text { Edad } \\
\text { (años) }\end{array}$ & $\begin{array}{l}\text { Talla } \\
\text { (CM) }\end{array}$ & Tanner & $\begin{array}{l}\text { Genitales } \\
\text { Externos }\end{array}$ & $\begin{array}{l}\text { Longitud de } \\
\text { Vagina (CM) }\end{array}$ \\
\hline $\mathbf{X}$ & 20.7 & 1.48 & II & $\begin{array}{l}\text { (2) Normales } \\
\text { (2) Hipoplásicos }\end{array}$ & 3.7 \\
\hline \multicolumn{6}{|c|}{ 1.A: Estudios paraclínicos } \\
\hline & Cariotip & $\begin{array}{l}\text { Ecografía } \\
\text { Ovario-útero }\end{array}$ & Urografía & $\begin{array}{l}\text { RX } \\
\text { Columna }\end{array}$ & $\begin{array}{l}\text { Laparoscopia } \\
\text { Gónada-útero }\end{array}$ \\
\hline $\begin{array}{l}\mathrm{X} \\
\text { 1A. } T\end{array}$ & $\begin{array}{l}46 \mathrm{XX} \\
\text { ratamier }\end{array}$ & AU. & AU. & Normal Normal & Band. AU. \\
\hline $\begin{array}{l}n=4 \\
\text { (2) } \\
\text { (2) }\end{array}$ & $\begin{array}{l}\text { Médico } \\
\text { Estróge } \\
\text { Neovag }\end{array}$ & $\begin{array}{l}\text { nos + Progestág } \\
\text { ina }+ \text { Terapia d }\end{array}$ & $\begin{array}{l}\text { ágenos cíclico } \\
\text { de suplencia }\end{array}$ & & \\
\hline
\end{tabular}

AU: Ausente

Band: Bandeleta.

En grupo 1B se ubican 12 pacientes con hipoplasia o agenesia úterovaginal secundaria a un pseudohermạfroditismo masculino y donde el estudio cromosómico mostró unà constitución 46XY. El motivo principal de consulta fue la ameno- 
rrea primaria $y / 0$ ambigüedad de genitales externos. Tabla No. 6.

Vale la pena aclarar que en este grupo se pueden presentar tres variantes: 1) Las que presentan feminización testicular completa, en las cuales se encuentra un fenotipo femenino con un desarrollo moderado de sus carácteres sexuales, hipoplasia de los dos tercios superiores de la vagina y agenesia del útero y trompas con testículos histológicamente normales (3 casos).

Tabla No. 6

1B: AUSENCIA CONGENITA DE VAGINA + DISGENESIA GONADAL XY

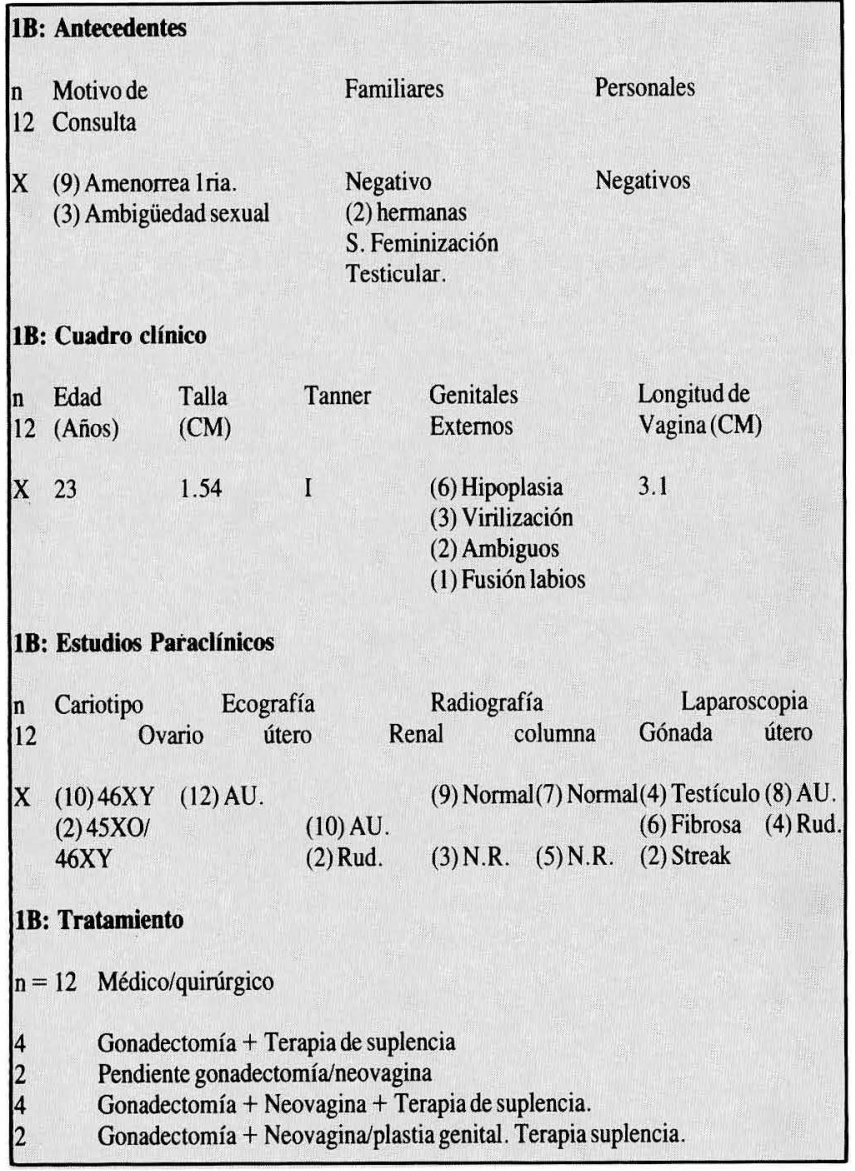

AU: Ausente

RUD: Rudimentario

N.R. No realizada

2) Síndrome de feminización testicular incompleta, en él encontramos un grado leve de virilización con hipoplasia de los dos tercios superiores de la vagina y agenesia del útero y trompas con tejido testicular también normal. ( 5 casos).

3) Pacientes con disgenesia gonadal XY en las cuales se encuentran grados diversos de virilización con hipoplasia vaginal y útero rudimentario (4 casos).

Se detectaron en este grupo dos casos de variedad mosaico $(45, \mathrm{XO} / 46, \mathrm{XY})$ y dos hermanas en el grupo de feminización testicular incompleta. No se detectaron otras malformaciones asociadas debido probablemente a que su origen etiológico es independiente de los ductos mullerianos. Su diagnóstico
Tabla No. 7

GRUPO II: AUSENCIA CONGENITA DE VAGINA/GONADA NORMAL -SINDROME DE MAYER-ROCKITANSKI KUSTER-HAUSER-

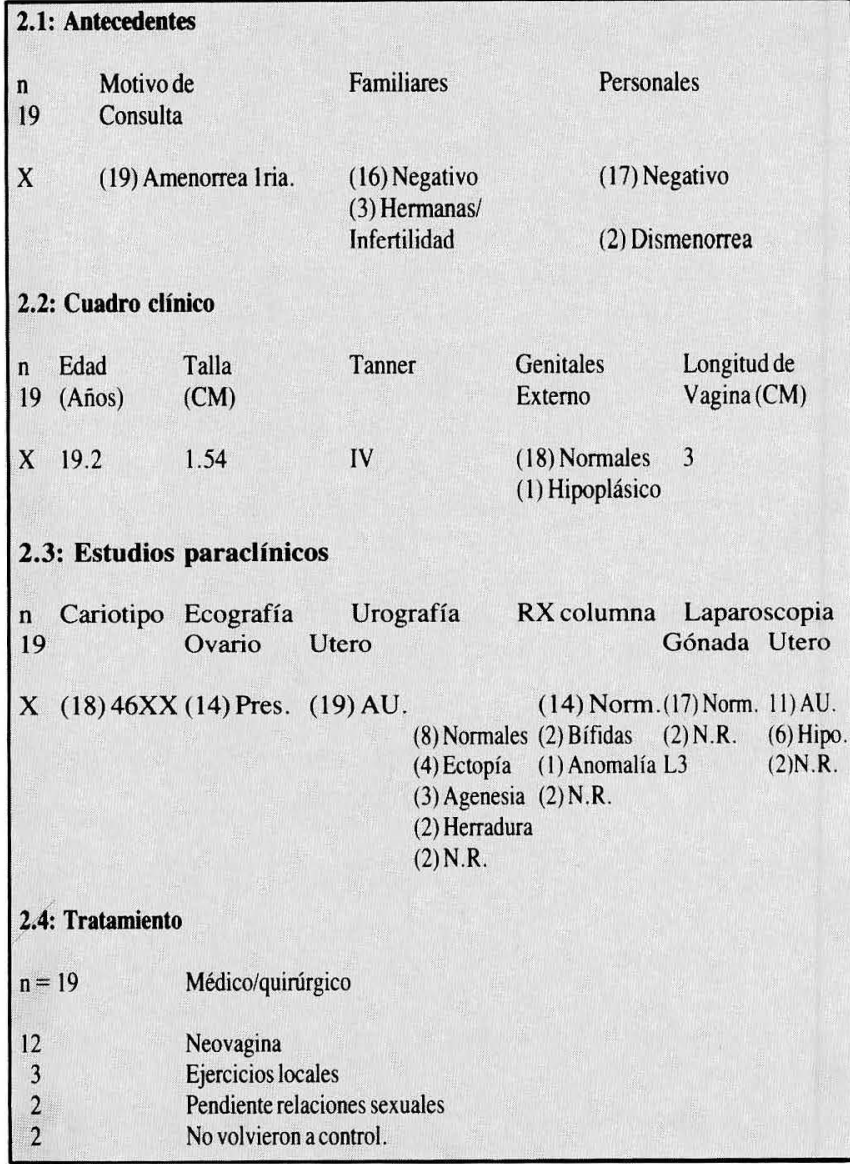

Pre: presente

AU: Ausente

Norm: Normal

Hipo: Hipoplásico

N.R.: No realizado

debe precisarse por laparoscopia y biopsia de la gónada. El manejo aceptado es la gonadectomía, reconstrucción vaginal y plastia genital en los casos de ambigüedad, más terapia de suplencia hormonal a base de estrógenos $(1.25 \mathrm{mg})$ y progestágenos $(10 \mathrm{mg})$ en forma cíclica.

En el grupo II se incluyen 19 pacientes que corresponden al clásico síndrome de Mayer-Rockitansky-Kuster Hauser. Clínicamente consultaron por amenorrea primaria, algunas con dolor cíclico, el desarrollo pondo-estatural normal, las características sexuales secundarias eran normales y al examen ginecológico llamó la atención el acortamiento de la vagina. El cariotipo fue normal y la ecografía mostró la presencia de ovario, ausencia o hipoplasia uterina que se confirmó por laparoscopia. La ecografía no es un método específico de diagnóstico, pues en cinco casos reportó ovarios ausentes y la laparoscopia informó su presencia. En este grupo llama la atención que 9 de 17 urografías realizadas (52\%) presentaban alteración renal (agenesia renal unilateral, riñón en herradura, ptosis). La radiografía de columna también demostró que 3 de 17 RX solicitadas (17\%) presen- 
taban alteraciones como espina bífida o lesiones a nivel de S1. Estos hallazgos corresponden a los informados por la literatura. El manejo es netamente quirúrgico y en algunos casos seleccionados se les pueden hacer ejercicios para distender este saco ciego ( 3 casos). El pronóstico obstétrico es nulo y sólo con las nuevas técnicas de fertilización asistida se les podrá practicar extracción de óvulos y brindar la posibilidad de una "madre sustituta". Tabla No. 7.

Las pacientes del grupo III están conformadas por aquellas que presentan alteraciones en la fusión de los ductos mullerianos. Es el más pequeño de la serie ( 3 casos); uno de ellos, útero bidelfo + agenesia de vagina. Y los otros dos con útero bidelfo y tabiques transversos vaginales. $\mathrm{La}$ amenorrea fue el síntoma principal y la oligomenorrea para uno de los casos que cursaba con tabique incompleto. El desarrollo pondoestatural fue normal y de nuevo encontramos un caso de 3 con agenesia renal derecha. El tratamiento quirúrgico en los casos de tabique es salvador pero la resección y plastia uterina, en los casos de útero didelfo o bidelfos, empobrece el pronóstico obstétrico. En casos de infección la histerectomía es la norma. Tabla No. 8

Tabla No. 8

AUSENCIA CONGENITA DE VAGINA CON ANOMALIAS DE LOS DUCTOS MULLERIANOS

\begin{tabular}{|c|c|c|c|c|c|}
\hline \multicolumn{6}{|c|}{ 3.1: Antecedentes } \\
\hline $\mathrm{N}=3$ & $\begin{array}{l}\text { Motivo de } \\
\text { de consulta }\end{array}$ & & Familiares & \multicolumn{2}{|c|}{ Personales } \\
\hline $\mathrm{X}$ & \multicolumn{2}{|c|}{$\begin{array}{l}\text { (2) Amenorrea 1ria. } \\
\text { (1) Oligomenorrea }\end{array}$} & Negativo & \multicolumn{2}{|c|}{ Dolor cíclico } \\
\hline \multicolumn{6}{|c|}{ 3.2: Cuadro clínico } \\
\hline$n=3$ & $\begin{array}{l}\text { Edad } \\
\text { (Años) }\end{array}$ & $\begin{array}{l}\text { Talla } \\
\text { (CM) }\end{array}$ & Tanner & $\begin{array}{l}\text { Genitales } \\
\text { Externos }\end{array}$ & $\begin{array}{l}\text { Longitud de } \\
\text { Vagina(CM) }\end{array}$ \\
\hline $\mathrm{X}$ & 23.6 & 1.52 & IV & Normales & 4.6 \\
\hline \multicolumn{6}{|c|}{ 3.3: Estudios paraclínicos } \\
\hline$n=3$ & Cariotipo & Ecografía & a Urografía & RX columna & Laparoscopia \\
\hline & & Ovario & Utero & & Gónada Utero \\
\hline $\mathrm{x}$ & $46 \mathrm{XX}$ & Prest. & $\begin{array}{l}\text { Prest.(2) Normales } \\
\text { (1) Agene.D. }\end{array}$ & $\begin{array}{l}\text { (2) Norm. } \\
\text { (1) Escolio- } \\
\text { sis. }\end{array}$ & $\begin{array}{l}\text { Norm. } 3 \text { Bidelf } \\
\text { (2) Tabique }\end{array}$ \\
\hline \multicolumn{6}{|c|}{ 3.4: Tratamiento } \\
\hline $\mathrm{n}=3$ & Quirúrgico & & & & \\
\hline $\mathrm{X}$ & \multicolumn{5}{|c|}{$\begin{array}{l}\text { (2) Resección tabique } \\
\text { (1) Histerectomía + neovagina }\end{array}$} \\
\hline
\end{tabular}

Prest: Presente

Norm: Normales

En el grupo IV se incluyen 8 pacientes con grados diversos de hipoplasia vaginal, agenesia de tercio inferior de vagina o himen imperforado, tabique transverso, útero normal, agenesia de cervix y gónadas normales. Se considera que las anomalías de estas pacientes corresponden a una alteración en la fusión del terminal caudal de los ductos mullerianos con el seno urogenital o anomalía en la diferenciación del seno urogenital.

El motivo de consulta fue la amenorrea primaria acompanada de color cíclico (dismenorrea), esta entidad es conocida en el pasado como criptomenorrea. El desarrollo físico fue normal así como las características sexuales secundarias. Por ser una alteración en la fusión del muller puede esperarse lesión renal (un caso de riñón en herradura). El estudio cromosómico reveló $46 \mathrm{XX}$ y un solo caso una variedad mosaico $46 \mathrm{XX} / 47 \mathrm{XXX}$. A nivel de vagina se pueden encontrar varias formas de obstrucción desde himen imperforado (1 caso), tabiques ( 2 casos).

Este grupo aunque se presenta como una urgencia debe disponer de un equipo multidisciplinario para analizar y preparar el tipo de cirugía, pues el drenaje sin una abertura permanente lleva a la infección, peritonitis y muerte, o en su defecto a una histerectomía ( 3 casos). Tabla No. 9

Tabla No. 9

GRUPO IV: AUSENCIA CONGENITA DE VAGINA/UTERO Y OVARIO NORMAL (TABIQUES LONGITUUINAL O TRANSVERSOS)

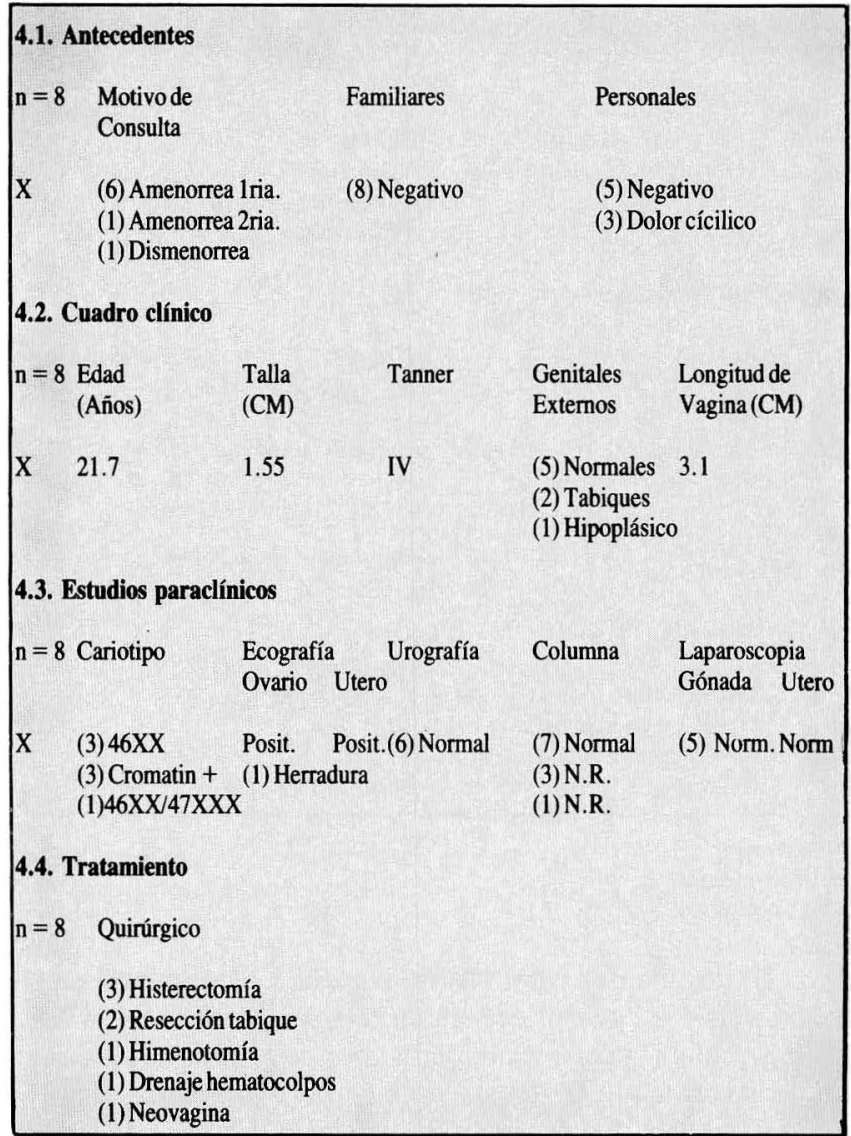

Posit: Positivo

N.R. No realizada.

En resumen del cuadro clínico observamos que el promedio de edad fue de 20 años y el motivo de consulta fue la amenorrea primaria excepto algunos casos del subgrupo IB que pueden consultar por ambigüedad sexual. Los antece- 
dentes tanto familiares como personales fueron negativos y sólo en los grupos III y IV acusaron dismenorrea. El exámen físico mostró que el desarrollo pondo-estatural era normal para nuestro medio y la hipoplasia de genitales sólo fue notoria en el grupo I. El acortamiento de vagina está disminuido en todos los grupos. Tabla No. 10.

Tabla No. 10

RESUMEN DE HALLAZGOS CLINICOS

\begin{tabular}{|llllllllll|}
\hline Grupo Edad & MOC & \multicolumn{2}{c|}{ Antecedentes } & \multicolumn{2}{c|}{ Talla } & Tanner G. Exter. & L. Vag \\
\hline & Años & \multicolumn{3}{c}{ Fami. } & Pers. & CM. & & CM. \\
IA & 20.7 & \multicolumn{2}{c}{ A. 1ria. } & $(-)$ & $(-)$ & 1.48 & II & Hipopla. & 3.7 \\
1B & 23.0 & Amb. Sex. $(-)$ & Herma. & 1.54 & I-II & Hipo/Viril. & 3.1 \\
II & 19.2 & A. 1ria. & $(-)$ & Infert. & 1.54 & IV & Normal & 3.0 \\
III & 23.6 & A. 1ria. & $(-)$ & Dismen. 1.52 & IV & Normales & 5.0 \\
IV & 21.7 & A. 1ria. & $(-)$ & Dismen. 1.55 & IV & Norm./Tabiq. 3.1 \\
\hline
\end{tabular}

Los exámenes paraclínicos muestran que el cariotipo es normal en todos los grupos exceptuando el IB con constitución cromosómica $X Y$. La ecografía orienta sobre la presencia o no de gónadas y útero, que debe ser confirmada por laparoscopia. La urografía y RX de columna confirman la asociación de lesiones renales y esqueléticas en fusiones del muller en los grupos II, III y IV. El tratamiento de la neovagina se realiza en todos los grupos, con cirugía complementaria como en el caso del grupo IB (gonadectomía), resección de tabique o histerectomía para el grupo III y IV si fracasa la plastia vaginal. Tabla No. 11.

Tabla No. 11

RESUMEN EXAMENES PARACLINICOS

\begin{tabular}{|c|c|c|c|c|c|c|}
\hline Grupo & C/tipo & Ecograf. & Urograf. & RX Colum. & Laparosco. & T/miento \\
\hline IA & $46 \mathrm{XX}$ & $(-)$ & Normal & Normal & Negat. & $\begin{array}{l}\text { Neovagi. } \\
\text { E2/Progs. }\end{array}$ \\
\hline IB & $\begin{array}{l}46 \mathrm{XY} \\
\text { Mosaico }\end{array}$ & $(-)$ & Normal & Normal & Gónada(+) & $\begin{array}{l}\text { Gonadect. } \\
\text { Neov,E2/Progs. }\end{array}$ \\
\hline II & $46 \mathrm{XX}$ & $\begin{array}{l}\text { Normal } \\
(-)\end{array}$ & Agenesia & $\begin{array}{l}\text { Normal } \\
\text { Bífida }\end{array}$ & $\begin{array}{l}\text { Ovario(+) } \\
\text { Utero(-) }\end{array}$ & Neovagina \\
\hline III & $46 \mathrm{XX}$ & Normal & Norm./Agene. & $\begin{array}{l}\text { Normal } \\
\text { Es/liosis }\end{array}$ & Didelfo & Neov. Tabiq. \\
\hline IV & $\begin{array}{l}46 \mathrm{XX} \\
\text { Mosaico }\end{array}$ & Normal & $\begin{array}{l}\text { Normal } \\
\text { Herradura }\end{array}$ & Normal & Normal & $\begin{array}{l}\text { Neov./tabiq. } \\
\text { Histerect. }\end{array}$ \\
\hline
\end{tabular}

\section{Discusión}

La ausencia congénita de vagina es una entidad que según los diferentes autores se presenta con una incidencia que varía entre 1:4.000 a 1:5.000 recién nacidas femeninas y 1:20.000 mujeres admitidas en un hospital general $(5,10$, $15,16,19)$.

Estos datos coinciden con los nuestros dado que encontramos 1-2 casos por año en un hospital de 15.000-20.000 admisiones por año (17).

Al revisar las diferentes clasificaciones sobre el tema como la de Evans (5), Buttran (19), Rock (20) no pudimos encasillar todos nuestros casos obligándonos a proponer una clasificación que por los datos encontrados es práctica tanto en su diagnóstico como en su manejo.

En el grupo IA, llama la atención los escasos reportes de la literatura sobre la asociación de ausencia congénita de vagina y disgenesia gonadal. (21-23). Se sabe por estudios experimentales y clínicos que el desarrollo de la gónada es independiente tanto del desarrollo del muller como del seno urogenital pero que éstos están influidos posteriormente por el estímulo estrogénico $(6,9)$. Genéticamente sabemos que alteraciones cromosómicas se encuentran asociadas a anomalías en los genitales como en el caso del Turner o del Klinefelter los cuales no se asocian con alteraciones en la diferenciación wolffiana o mulleriana. Recientemente, se ha reportado en la literatura un síndrome de disgenesia gonadal pura (XX) asociado a anomalías esqueléticas (Síndrome Murcs) (24).

Su diagnóstico debe basarse en el examen clínico, cariotipo y laparoscopia. La extirpación de la gónada ofrece controversia ante la descripción de gonadoblatoma $25 \%$ en algunos casos de "streak gonadal" (25). Estas pacientes son estériles, su pronóstico obstétrico es nulo, y el tratamiento debe ser plastia vaginal al iniciar relaciones sexuales y terapia de suplencia con estrógenos y progestágenos hasta los 50-60 años.

En el grupo I incluimos un subgrupo B, compuesto por aquellos casos de pseudohermafroditismo masculino los cuales a pesar de no hacer parte de la clasificación clásica, son pacientes que consultan por amenorrea, genitales ambiguos y al demostrar su cariotipo XY deben someterse a una gonadectomía, reconstrucción de genitales externos o plastia vaginal. (15-17). En este punto vale la pena considerar que si no se hace el diagnóstico después del nacimiento y su formación es de varón, al llegar a la adolescencia se practica plastia escrotal y terapia a base de andrógenos, si por el contrario la formación fue femenina, se debe practicar gonadectomía pues en el $20 \%$ puede aparecer una neoplasia (seminoma) $(21,25)$. Para aquellos casos que consultaron tardíamente y donde había crecimiento del glande (feminización testicular incompleta), se realizará una clitoridioplastia y no sección como se preconizaba hace algunos años. La terapia secuencial con estrógenos y progestágenos se impone para evitar todas las complicaciones del hipoestrogenismo.

El grupo II es el más representativo y el que más atención ha recibido por los diferentes grupos de investigación en este campo. $(5,10,12,15,16,17,20,30,36,44,60)$. Los datos de la presente investigación coinciden con los aparecidos en la literatura donde se encuentra una asociación de $34 \%$ de anormalidades urinarias y $12 \%$ esqueléticas $(5$, $10,20)$.

Dado que el síndrome se caracteriza por amenorrea primaria, cariotipo $46 \mathrm{XX}$, útero que varía desde hipoplásico hasta un vestigio, desarrollo de las características sexuales secundarias normales, función ovárica normal y asociación con anormalidades renales, esqueléticas o cardíacas (29, 31-34). El estudio debe incluir cariotipo, ecografía pélvica, urografía, RX, tórax/columna cervical, laparoscopia y en el futuro resonancia nuclear magnética (35). El futuro obstétrico de este grupo es nulo y sólo los programas de ferti- 
lización asistida para el grupo II y la microcirugía para el grupo III pueden ser esperanzadoras. El apoyo psicológico debe ser constante en todos estos grupos (61).

El grupo III aunque escaso es el más complicado pues se presentan las diferentes anormalidades del muller, desde el útero didelfo, bicorne, unicorne, septado, asociado a anormalidades de vagina desde himen imperforado hasta tabiques longitudinales o transversos (44-47). Como se sabe las lesiones del muller se asocian a anormalidades renales y esqueléticas $(36,40,49,50)$. En nuestra escasa estadística tenemos un caso de tres con lesión renal y otro con lesión esquelética. El pronóstico obstétrico depende del grado de lesión uterina y si quirúrgicamente no es posible recuperar el útero se recomienda la plastia vaginal e histerectomía.

$\mathrm{El}$ grupo IV presenta útero normal y el III útero anormal. Estos dos grupos por tener útero y al no poder salir la sangre menstrual presentan clínicamente dolor cíclico y llegan a la consulta como una urgencia. $(36,39,44,46)$. El separarlos en grupos diferentes obedece en primer lugar a que en el grupo III hay variantes como la agenesia de cervix descrita por otros autores $(19,42)$. Tabiques transversos o longitudinales $(5,20,39)$. Su manejo dada las complicaciones al hacer drenaje del hematometra/hematosalinx ha sido la histerectomía pues la mayoría de estos casos terminan en sepsis. Recientemente se describe la plastia vaginal con un tubo endotraqueal con buenos resultados (41). Nosotros hemos intentado esta técnica pero desafortunadamente ha habido estenosis de la plastia a pesar de las dilataciones proponiendo como técnica quirúrgica practicar unas " $Z$ " en el sitio de la estenosis. En este grupo Rock (20) describe embarazos exitosos en estos casos.
Para el manejo de la agenesia de vagina son variadas las propuestas de tratamiento desde la descripción de Heppner en 1872 (51) usando labio mayor como injerto, Abbe 1898 usando injertos de piel (52) y posteriormente popularizada dicha técnica por McIndoe en el 37 (53). Posteriormente se utilizaron colgajos de recto Coway (54), colon (59), íleon por Baldwin en 1904 (55), éstas últimas abandonadas por sus efectos secundarios. Recientemente se ha propuesto el uso de amnios (61) pero los resultados no son alentadores. Los diferentes estudios muestran que la técnica de injerto libre de piel da buenos resultados (56-58). Los injertos pueden a veces presentar mal olor y la resequedad mientras se reepitelizan (64). Dentro de los tratamientos no quirúrgicos están el uso de dilatadores de Hegar, de plástico y el uso de la bicicleta de Ingram (62-63) que acoplada a un dispositivo/dilatador en el galápago produce dilatación progresiva de la vagina. En nuestra experiencia el injerto libre de piel en asocio con dilatador de plástico nos ha dado buenos resultados, a pesar que en 3 de 13 casos se presentaron esténosis de la plastia.

Si la paciente es diagnosticada en el período infantil se deja llegar a la edad reproductiva y ante la posibilidad del inicio de relaciones sexuales se propone la plastia vaginal (grupos I y II). En este punto hay algo de controversia con los cirujanos pediatras quienes proponen la realización de la neovagina una vez diagnóstica. La ejecución de esta cirugía implica un equipo compuesto por ginecólogos, urólogos, cirujanos plásticos, psicólogos. El objetivo fundamental es brindarle una mejor calidad de vida a este grupo de mujeres que presentan este defecto (65-66).

\section{BIBLIOGRAFIA}

1. Mayer CAJ. Uber verdoppelungen des uterus und inre artennebst bemerkungen uber hasenscharte und wolfsrachen. J. Chir Auger 1829; 13: 525-564.

2. Rockitansky $\mathbf{K}$. Uber die sogenannten verdoppelungen des uterus Med. JB. Ost. Staat. 1838; 26: 37-39.

3. Kuster H. Uterus bipartitus solidus rudimentarius cum vagina solida. Z. Gen. Gyn. 1910; 67: 692-718.

4. Hauser GA., Schereiner WE., Das Mayer-Rockitansky-Kuster. Syndrom Schweiz Med. Wochenschr 1961; 91: 381-384.

5. Evans TN., Poland ML. Vaginal malformations. AM. J. Obstet. Gynecol. 1981; 141: 910-920.

6. Carson SA., Simpson SL. et al. Heritable aspects of uterine anomalies II. Genetic analysys of mullerian aplasia. Fertil Steril 1983;40: 1-4.

7. David A., Carmil BA. Congenital abscence of the vagina. Clinical and psychologic aspects. Obstet. Gynecol. 1975; 46: 4-7.

8. Elias S., Simpson JL. et al. V genetics studies in incomplete mullerian fusion. Obstet. Gynecol. 1984; 63: 3-5.

9. Fores. Hammond C. et al. Urologic and genital anomalies in patients with congenital abscence of the vagina. Obstet. Gynecol. 1975; 46: 4-6.

10. Griffin J., Edwards C. et al. Congenital abscence of the vagina. The Mayer-Rockitansky-Kuster-Hauser. Ann. Intern. Med. 1976; 85: 224-236.

11. Grover S., Solanki BR. A clinical pathologic study of mullerian duct aplasia with special reference to cytogenetics studies. Am. J. Obstet. Gynecol. 1970; 107: 1-5.

12. Jones $\mathbf{H}$., Hermut $\mathrm{S}$. Familial ocurrence of congenital abscence of the vagina. Am. J. Obstet. Gynecol. 1972; 114: 8-10.

13. Simpson JL. Genes and chromosomes that cause female infertility. Fertil Steril 1985; 44: 6-9.
14. Verf M., Simpson JL. et al. Heritable aspects of uterine anomalies. I three familial agregates with mullerian fusion anomalies. Fertil Steril 1983; 40: 1-3.

15. Jaffe RB. Disorders of sexual development. En yen SC. Jaffe RB. Reproductive endocrinology, 2th edition. W. Saunders. Co. 1986; 283.

16. Mishell DR. Disorders of sexual differentiation. En Mishell Dr. Davajan V. Reproductive Endocrinology Infertility and contraception. Davis Co. Philadelphia 1986; 191.

17. Williams RH. Tratado de endocrinología 5th. Edit. Interamericana 1984.

18. Artega C., Onatra W. Alteraciones de diferenciación sexual. Curso complicaciones médico-quirúrgicas en ginecología. Universidad Nacional de Colombia. Edit. Presencia Bogotá. 1989; 13-18.

19. Buttram VC. Reiter surgical treatment of the infertile female Williams \& Wilkins. Baltimore 1985; 149-199.

20. Jones HW., Rock JA. Reparative and constructive surgery of the female generative tract. Williams. Baltimore 1983; 146-185.

21. Phansey SA., Tsai CC. Vaginal agenesis in association with gonadal dygenesis. Obstet. Gynecol. 1981; 57: 56S-57S.

22. De RC. Mitra J. Mitra AK. Vaginal agenesis and the gonads. J. Indian Med. Assoc. 1981; 77: 105-109.

23. Gondos B. Diagnosis of abnormalities in gonadal development. Ann. Clin. Lab. Sci. 1982; 12: 276-287.

24. Mendez JP., Ulloa-Aguirre A. y col. Endocrine evaluation in a patient with murcs association and ovarian agenesis. Eur. J. Obstet. Gynecol. Reprod. Biol. 1986; 22: 161-169.

25. Gunnala S., Eskin B. Pure gonadal dysgenesis with microscopic ovarian streak and gonadoblastoma. Obstet. Gynecol. 1981; 57: 58S-61S. 
26. Madam K., Schoemaker J. XY females with enzyme deficiencies of steroid metabolism. Hum. Genet. 1980; 53: 291-295.

27. Troche V., Hernandez E. Neoplasia arising in dysgenetic gonads. Obstet. Gynecol. Surv. 1986; 41: 74-79.

28. Migeon CJ. Male pseudohermafrophroditism. Ann. Endocrinol 1980; 41: 311-343.

29. Guthrie R., Buggeln J., Rockitansky syndrome: report of two cases. J. Am. Osteopath. Assoc. 1981; 80: 344-347.

30. Tarry MF. Ducket JW. Stephens FD. The mayer rockitansky syndrome: pathogenesis, classification and management. J. Urol 1986; 136: 648-652.

31. Ghirardini G., Segre A. Vaginal agenesis (Mayer Rockitansky-Kuster Hauser Syndrome): recent atiopathogenetical and anatomical views. Clin. Exp. Obstet. Gynecol. 1982; 91: 98-102.

32. Vasquez SB. Mullerian duct agenesis and other congenital anomalies. J. Adolesc Health Care 1982; 2: 289-290.

33. Interaprasert S., Benchakan V. Mullerian duct anomalies and unilateral renal agenesis. J. Med. Assoc. Thai. 1985; 68: 183-189.

34. Aci'en P. Armi'nana E. Unilateral renal agenesis associated with ipsilateral blind vagina. Arch. Gynecol. 1987; 240: 1-8.

35: Togashi K., Nishimura K. y col. Vaginal agenesis: classification by magnetic resonance imagin. Radiology 1987; 162; 675-677.

36. Rock JA., Baramki TA. y col. A unilateral functioning uterine anlage with mullerian duct agenesis. Int. J. Gynecol. Obstet. 1980; 18: 99-101.

37. Riggall FC., Cantor B. 46XX hernia inguinale and vaginal agenesis. Obstet. Gynecol. 1980; 56: 265-266.

38. Jal'uvka V. Vaginal discharge the only symtons of uteri communicantes. Zentralbl Gynecol. 1980; 102: 1448-1452.

39. Pinsonneault O., Goldstein DP. Obstructing malformations of the uterus and vagina. Fertil Steril 1985; 44: 241-247.

40. Bernhisel MA., London SN. Unusual mullerian anomalies associated with distal extremity abnormalities. Obstet. Gynecol. 1985; 65: 291-294.

41. Bates GW., Wiser WL. A technique for uterine conservation in adolescents with vaginal agenesis and a functional uterus. Obstet. Gynecol. 1985; 66: 290-294.

42. Marklam SM., Parmley TH. y col. Cervical agenesis combined with vaginal agenesis diagnosed by magnetic resonance imaging. Fertil Steril 1987; 48: 143-145.

43. Wakabayashi A., Fujji H. y col. A case of hydronetrocolpos with left renal agenesis. Hinyokika Kiko 1985; 31: 1635-1641.

44. Rock JA., Jones HW JR. The double uterus associated with an obstructed hemivagina and ipsilateral renal agenesis. Am. J. Obstet. Gynecol. 1980; 138: 339-342.

45. Eisenberg E., Farber M. y col. Complete duplication of the uterus and cervix with a unilaterally imperforate vagina. Obstet. Gynecol. 1982; 60: 259-262.

46. Miyazak Y., Ebisuno S. y col. Uterus didelphis with unilateral imperforate vagina and ipsilateral renal agenesis. J. Urol. 1986; 135: 107-109.
47. Rosenberg KH., Udassin R. y col. Duplication of the uterus and vagina, unilateral hidrometrocolpos and ipsilateral renal agenesis: sonographic aid to diagnosis. J. Ultrasound. Med. 1982; 1: 289-291.

48. Deutsch M., Beck D. y col. Uterus duplex with a unilateral imperforate vagina, diagnosis and treatment. Ann. Chir. Gynecol. 1985; 74: 247-249.

49. Felici A., Cappellano E. y col. A case of vaginal agenesis with rudimentary uterine horns and labio-papatal schisis. Minerva Gynecol. 1985; 37: 501-505.

50. Reece EA., Husami N., Baxi L. A succesful gestational outcome in the presence of genitourinary abnormalities and severe medical complications of pregnancy. Diag. Gynecol. Obstet. 1982; 4: 347-349.

51. Heppner (1872). Citado por Paunz A. Formation of an artificial vagina to remedy a congenital defect. Zen Fur Gyn. 1923; 47: 833-836.

52. Abbe R. New method of creating a vagina in a case of congenital absence. Med. Recor. 1898.

53. McIndoe A. The aplication of cavity grafting. Surgery 1937; 1:535-537.

54. Coway H., Stark RB. Construction and reconstruction of the vagina. Surg. Gyn. Obstet. 1953; 97: 573-575.

55. Baldwin If. The formation of an artificial vagina by intestinal transplantation. Annals Surg. 1904; 40: 398-400.

56. Wilflingseder PI. Management of vaginal agenesis. Congenital malformations. Excerpta medica. Amsterdam-Oxford 1980.

57. Marshall FF. Vaginal agenesis. Clin. Plast. Sur. 1980; 7: 175-178.

58. Karjalainen O., Myllynem L. Management of vaginal agenesis. Ann. Chir. Gynecol. 1980; 69: 37-41.

59. Novak F. Use of the colon in the surgical treatment of vaginal agenesis. Gynakologe 1980; 13: 122.

60. De Souza Az., Maluf M. Surgical treatment of congenital utero-vaginal agenesis: Mayer Rockitansky-Kuster-Hauser. Syndrome. Int. Surg. 1987; 72: 45-47.

61. Ashwoth MF., Morton KE. y col. Vaginoplasty using amnion. Obstet. Gynecol 1986; 67: 443-446.

62. Ingram JM. The bicycle seat stool in the treatment of vaginal agenesis and stenosis: a preliminary report. Am. J. Obstet. Gynecoi. 1981; 140 : 867-873.

63. Williams JK., Lake M., Ingram JM. The bicycle seat stool in the treatment of vaginal agenesis and stenosis. J. Obstet. Gynecol. Neonatal Nurs 1985; 14: 147-150.

64. Herman CJ., Willemsen WN. y col. Artificial vaginas: possible sources of epithelialization. Hum Pathol 1982; 13: 1100-1105.

65. Raboch J., Horejsi J. Sexual life of women with the Kuster-Rockitansky Syndrome. Arch. Sex. Behav. 1982; 11: 215-220.

66. Poland ML., Evans TN. Psychologic aspects of vaginal agenesis. J. Reprod. Med. 1985; 30: 340-344.

67. Alan LM. Vaginal Reconstruction. Clin. Obstet. Gynecol. 1985; 12: 515-525. 